\title{
Future Outlook of Checkpoint Receptor Inhibitors
}

\author{
Nahla AM Hamed* \\ Department of Hematology, Faculty of Medicine, Alexandria University, Egypt
}

Submission: December 18, 2017; Published: January 23, 2018

*Corresponding author: Nahla AM Hamed, Department of Clinical Hematology and Bone Marrow Transplant, Faculty of Medicine, Alexandria University, Egypt, Email: drhamedn@hotmail.com

\begin{abstract}
Numerous immune checkpoints inhibitors have been identified to date, and are in varying stages of pre-clinical and clinical development. It is essential that their common adverse effects become well versed. The main problem with checkpoint blockers is that only a fraction of patients respond to therapy. Different immune checkpoints regulate different stages and cell types of the immune response. Combining checkpoint targeting treatments will likely improve clinical response rates. Immune checkpoints may also be used in combination with other immune therapies such as cancer vaccine to augment the immune response.

Abbreviation: CTLA-4: Cytotoxic T-Lymphocyte-Associated protein-4; PD-1: Programmed Cell Death Protein-1; TIM-3: T-Cell Immunoglobulin-3; LAG-3: Lymphocyte Activation Gene-3; irAE: Immune-Related Adverse Events; CRS: Cytokine Release Syndrome; ICPIs: Immune Checkpoints Inhibitors
\end{abstract}

\section{Introduction}

$\mathrm{T}$ cells are major contributors of the antitumor immune response. A major determinant of their ability to generate clinically meaningful responses is dictated by the effective engagement of the $\mathrm{T}$ cell with its target. This interaction is regulated by a complex balance of co-stimulatory and coinhibitory bi-directional signals whose physiologic role is the maintenance of self-tolerance and prevention of autoimmunity [1]. These immune checkpoint molecules also stimulate immune responses against tumor cells [2]. In a cancer patient, tumor cells can hijack the expression of immune checkpoint molecules to evade immunologic destruction [3]. Numerous immune checkpoints have been identified to date, and are in varying stages of pre-clinical and clinical development [4]. The most commonly targeted checkpoints for cancer immunotherapy are CTLA-4, PD-1, TIM-3 and LAG-3 [2]. Ipilimumab (CTLA-4 blocker), nivolumab and pembrolizumab (two PD-1 blockers) and atezolizumab, avelumab and durvalumab (three PD-L1 blockers) are approved for the treatment of different solid tumors such as melanoma, lung cancer, head and neck cancer, bladder cancer and meckel cell cancer as well as hematological tumors such as classic Hodgkin lymphoma [2]. They have been incorporated into therapeutic protocols for first-second- or third-line agents for these metastatic cancers [5]. Only a subset of cancers currently show favorable response rates to checkpoint immunotherapy, in part due to varying immunogenicity of different tumors and immunosuppressive tumor microenvironments. Pancreatic cancer is a highly lethal cancer that has been shown to have a dearth of tumor-infiltrating effector T cells, and which has failed to respond to single-agent checkpoint inhibitors [4]. Given the current and increasing indications for these drugs, it is essential that their common adverse effects become well versed and other less documented clinical conditions that could be unmasked with the use of such medications be observed [5]. An understanding of these newly-emerging immune checkpoints will open the door to different combination therapies that may better modulate the immune system to promote an anti-tumor response and limit auto-immune related side effects [4].

\section{Cancer immunotherapy associated risks}

Risks associated with cancer immunotherapy can be broadly classified into auto immune toxicity and cytokine-associated toxicity [6]. Importantly, trials comparing PD-1-directed therapy vs. CTLA-4-directed therapy, demonstrate fewer immunerelated adverse events in patients receiving anti-PD-1 treatment [4]. Anti-CTLA-4 and anti-PD-1 combination therapy has demonstrated improved clinical response rates in melanoma compared to either agent alone [4]. However, the combination of ICPI ipilimumab and nivolumab recently approved for the treatment of advanced melonoma is associated with a more severe side effect profile compared with the agents used as monotheray [7].

\section{Autoimmune toxicity}

Therapy with CTLA-4 inhibitors and PD-1 and PD-L1 blocking agents are frequently associated with wide spectrum 
of irAE [8]. Also-called "on target, off-tumor toxicity," they result from antigen-specific attack on host tissues when the targeted tumor associated antigen is expressed on nonmalignant tissue [6]. The following are reported side effects.

\section{Cardiac toxicity}

Cardiac toxicity has been underestimated. Several cases of fatal heart failure have been documented in melanoma patients treated with checkpoint inhibitors during the last years since PD-1 and PD-L1 can be expressed in human cardiomyocytes [8].

\section{Endocrine irAE}

Including hypophysitis and thyroiditis has been reported during treatment with monoclonal antibodies against CTLA4 [9].

\section{Renal effects}

Acute (granulomatous) tubulointerstitial nephritis and immune complex glomerulonephritis have been identified. Acute renal damage can be reversed upon ICPIs drug discontinuation and introduction of systemic corticosteroid treatment. Any delay in treating this complication could result in definitive and irreversible renal injury [10].

\section{Cutaneous side effects}

Are often dose limiting and can lead to discontinuation of therapy. Ipilimumab induce morbilliform eruption on the trunk and extremities and pruritis (most commonly), toxic epidermal necrolysis, severe drug rash with eosinophilia and systemic symptoms, Sweet syndrome and cutaneous sarcoidosis. The cutaneous events usually occur early in the course of the disease and are dose dependent. PD-1 inhibitors (nivolumab and pembrolizumab) induce toxicities similar to CTLA-4 but are less severe and of later time of onset. The most common cutanous adverse events include lichenoid reactions, eczema, vitiligo and pruritis. Lichenoid oral mucosal lesions are located on the tongue, buccal mucosa, lips or gingivae or located on all of these. Anti-PD-L1 antibodies such as atezolizumab have similar side effect profile compared with the PD-1 inhibiors [7].

\section{Neurological}

irAEs are rare complication of ICPIs that can lead to long-term morbidity. A rare case of encephalopathy after pembrolizumab treatment was reported. Early data suggest that neurological irAEs correlate with a favorable disease response. Early administration of high-dose corticosteroids and cessation of ICPI therapy is often necessary after grade 3 or 4 irAEs [11].

\section{Pulmonary irAEs}

Pneumonitis is a potentially fatal toxicity of anti-PD-1/PDL1 ICPI. Its incidence rate in advanced solid tumors is estimated at $5 \%$ with $1.8 \%$ are grade $3+$ events. Clinically, patients may present with dyspnea, cough, fever and chest pain; or may be asymptomatic with radiologic findings alone. Its mechanism of development is unknown. Pneumonitis resolves clinically with corticosteroid therapy alone in the majority of cases. A subset of patients requires additional immunosuppressive medications. Patients who clinically improved with steroid treatment must be monitored closely in the outpatient setting. If pneumonitis management results in complete clinical and radiological resolution, patients may be able to restart their ICPI therapy. It is currently unclear which population of patients is more susceptible to developing high grade or steroid-refractory pneumonitis [3].

\section{Autoimmune hemolytic anemia}

A definite association between autoimmune hemolytic anemia and nivolumab has not been clearly documented, although a few cases of autoimmune hemolytic anemia have been reported recently. A fatal case of autoimmune hemolytic anemia refractory to steroids in a patient treated with nivolumab for metastatic lung cancer has been reported [5].

\section{Cytokine-associated toxicity}

Also known as CRS, it is a non-antigen specific toxicity that occurs as a result of high-level immune activation. Large numbers of lymphocytes and/or myeloid cells become activated and release inflammatory cytokines. IL-6 is implicated as a central mediator of CRS toxicity. TNF and IL-1 may also contribute. Fever is a hallmark. It may exceed $40.0^{\circ} \mathrm{C}$. MRI often reveals no abnormalities. Complications include severe cardiac dysfunction (typically reversible), adult respiratory distress syndrome, varied neurologic toxicity, renal and/or hepatic failure, and disseminated intravascular coagulation. Tocilizumab is an effective treatment for severe or life-threatening CRS. It prevents IL-6 binding to cell-associated and soluble IL-6Rs. If the patient's condition does not improve or stabilize within 24 hours of tocilizumab dose, administration of a second dose of tocilizumab and/or a second immune suppressive agent, such as corticosteroids, should be considered. Dexamethasone may be given in severe neurologic symptoms. Anti-TNF mAbs (infliximab), soluble TNF receptor (etanercept) and IL-1R-based inhibitors (anakinra) could provide benefit [8].

\section{Drug resistance}

The main problem with checkpoint blockers is that only a fraction of patients respond to therapy [2]. Although inhibitors of CTLA-4 and PD-1/ PD-L1 pathway can unleash anti-tumor immunity and mediate cancer regression [8], a substantial proportion of initial responders ultimately relapse with lethal, drug resistant disease months or years later. Studies have shed light on the rich functional landscape of mutations that endow tumor cells with the ability to evade T-cell-mediated immune surveillance. Cancer genomes bear signatures of clonal evolution and selection particularly implicating acquired defects in interferon receptor signaling and antigen presentation [12].

\section{Future Outlook}

The future of cancer immunotherapy is very promising [4]. Clinical anti-tumor response should improve by simultaneously 
blocking CTLA-4 and PD-1 as their inhibitory signals will be blocked at multiple steps and in multiple cell types. CTLA4 is thought to play a role pre-dominantly in regulating the magnitude of early $\mathrm{T}$ cell activation, while PD-1 is expressed upon $\mathrm{T}$ cell activation and regulates effector $\mathrm{T}$ cell activity. Furthermore, CTLA-4 expression is restricted to only $\mathrm{T}$ cells, while PD- 1 is found on T cells, B cells and NK cells. In addition, the combination of ICPI with neo antigen vaccines could offer a new approach for optimizing anti-tumor $\mathrm{T}$ cell immunity. Vaccine immunotherapy offers the possibility of priming the immune system to tumor antigens, allowing for a more robust response to checkpoint inhibitors [4].

\section{Conclusion}

Combination immune checkpoint therapy and combination of anti-tumor vaccine therapy with immune checkpoint therapy are both promising methods to improve response rates and broaden the use of immunotherapy across different tumor types.

\section{References}

1. Hoyos V, Borrello I (2016) The immunotherapy era of myeloma: monoclonal antibodies, vaccines and adoptive T-cell therapies. Blood 128(13): 1679-1687.

2. Rotte A, Jin JY, Lemaire V (2017) Mechanistic overview of immune checkpoints to support the rational design of their combinations in cancer immunotherapy. Ann Oncol doi:10.1093/annonc/mdx686.

3. Balaji A, Verde F, Suresh K, Naidoo J (2017) Pneumonitis from antiPD-1/PD-L1 therapy. Oncology (Williston Park) 31(10): 739-746, 754.
4. Torphy RJ, Schulick RD, Zhu Y (2017) Newly emerging immune checkpoints: promises for future cancer therapy. Int J Mol Sci 18(12): 2642.

5. Palla AR, Kennedy D, Mosharraf H, Doll D (2016) Autoimmune hemolytic anemia as a complication of nivolumab therapy. Case Rep Oncol 9(3): 691-697.

6. Lee DW, Gardner R, Porter DL, Louis CU, Ahmed N, et al. (2014) Current concepts in the diagnosis and management of cytokine release syndrome. Blood 124(2): 188-195.

7. Collins LK, Chapman MS, Carter JB, Samie FH (2017) Cutaneous adverse effects of the immune checkpoint inhibitors. Curr Probl Cancer 41(2): 125-128.

8. Varricchi G, Marone G, Mercurio V, Gaidiero MR, Bonaduce D, et al. (2017) Immune checkpoint inhibitors and cardiac toxicity: an emerging issue. Curr Med Chem doi:10.2174/0929867324666170407125017.

9. Cap J (2016) Endocrine complications of modern cancer therapy. Vnitr Lek 62(9 Suppl 3): 45-69.

10. Izzidine H, Mateus C, Boutros C, Robert C, Rouvier P, et al. (2017) Renal effects of immune checkpoint inhibitors. Nephrol Dial Transplant 32(6): 936-942.

11. Feng S, Coward J, McCaffery E, Coucher J, Kalokerinos P, et al. (2017) Pembrolizumab-induced encephalopathy: a review of neurological toxicities with immune checkpoint inhibitors. J Thorac Oncol 12(11): 1626-1635.

12. Syn NL, Teng MYL, Mok TSK, Soo RA (2017) De-novo and acquired resistance to immune checkpoint targeting. Lancet Oncol 18(12): e731-e741.

\section{Your next submission with Juniper Publishers} will reach you the below assets

- Quality Editorial service

- Swift Peer Review

- Reprints availability

- E-prints Service

- Manuscript Podcast for convenient understanding

- Global attainment for your research

- Manuscript accessibility in different formats

( Pdf, E-pub, Full Text, Audio)

- Unceasing customer service

Track the below URL for one-step submission https://juniperpublishers.com/online-submission.php 